\title{
Evaluación in vitro de Dos Fármacos de Uso Veterinario frente a Patógenos Causantes de Otitis Externa en Perros
}

\author{
In vitro Evaluation of Two Drugs of Veterinary Use Against Pathogens Causing \\ Otitis Externa in Dogs
}

\author{
Luz Adriana Gutiérrez R. ${ }^{1,2,3}$, Carolina Ortiz del Río ${ }^{1}$, Juan Esteban Hincapié1, \\ Luz Adriana Ramírez A. ${ }^{1}$
}

\section{Resumen}

\begin{abstract}
El objetivo del presente estudio fue evaluar el efecto in vitro de dos fármacos de uso veterinario frente a microorganismos aislados postratamiento en 27 perros con otitis externa. Se aislaron dos cepas de Pseudomonas aeruginosa, tres de Staphylococcus intermedius y tres levaduras del género Malassezia de cinco perros. El nivel de efectividad de los antimicrobianos se determinó por el método de Concentración Inhibitoria Mínima (CIM). Se prepararon soluciones de gentamicina $(3 \mathrm{mg} / \mathrm{ml})$ y de clotrimazol (10 $\mathrm{mg} / \mathrm{ml})$. Las bacterias y levaduras se llevaron a una concentración de 1x10 microorganismos $/ \mathrm{ml}$. En las placas de ELISA se colocó $150 \mu \mathrm{l}$ de cada bacteria o levadura y $150 \mu \mathrm{l}$ de gentamicina o clotrimazol. Se hicieron dos repeticiones y tres réplicas de cada microorganismo. Para los controles, se utilizaron los fármacos más agua y microorganismos más agua, ajustados a $300 \mu \mathrm{l}$ c/u. Las condiciones de crecimiento en el espectrofotómetro fueron de $35^{\circ} \mathrm{C} / 10 \mathrm{rpm} / 24 \mathrm{~h}$, donde cada $2 \mathrm{~h}$ se midió la cinética de crecimiento del microorganismo a 660 de densidad óptica (DO). Se encontró que la gentamicina, en una dosis de $3 \mathrm{mg} / \mathrm{ml}$, produce una inhibición del crecimiento adecuado de las bacterias, en tanto que el clotrimazol podría emplearse en el caso de otitis causadas por levaduras $(M$. pachidermatis), pero en concentraciones superiores a $14 \mathrm{mg} / \mathrm{ml}$.
\end{abstract}

Palabras clave: antimicrobiano, antifúngico, antibiótico, sensibilidad

\section{Abstract}

The aim of this study was to evaluate the in vitro effect of two drugs of frequent use in veterinary practice against microorganisms isolated after treatment in 27 dogs with otitis externa. Various isolates were obtained from five dogs: two Pseudomonas aeruginosa, three Staphylococcus intermedius, and three yeasts of the genus Malassezia. The level of effectiveness of the antimicrobial drugs was determined by the method of

\footnotetext{
${ }^{1}$ Corporación Universitaria Lasallista, Antioquia, Colombia

${ }^{2}$ E-mail: lugutierrez@lasallistadocentes.edu.co

${ }^{3}$ Proyecto financiado por el Fondo para la Investigación Corporación Universitaria Lasallista
}

Recibido: 4 de diciembre de 2013

Aceptado para publicación: 19 de agosto de 2014 


\begin{abstract}
Minimum Inhibitory Concentration (MIC). Gentamicin $(3 \mathrm{mg} / \mathrm{ml})$ and clotrimazole $(10 \mathrm{mg} / \mathrm{ml})$ solutions were prepared. Bacteria and yeast were brought to a concentration of $1 \times 10^{6}$ microorganism $/ \mathrm{ml}$. In ELISA plates, $150 \mu \mathrm{l}$ of each bacteria or yeast and $150 \mu \mathrm{l}$ of gentamicin or clotrimazole was added. Two repetitions and three replicates of each microorganism were considered in the study. As controls, drugs plus water and microorganisms plus water were used, and the volume adjusted to $300 \mu \mathrm{l}$ each. Growth conditions in the spectrophotometer were $35{ }^{\circ} \mathrm{C} / 10 \mathrm{rpm} / 24 \mathrm{~h}$, where the growth kinetics of the microorganisms were measured at $660 \mathrm{OD}$. Results showed that gentamicin at a dose of $3 \mathrm{mg} / \mathrm{ml}$ causes an adequate growth inhibition of the bacteria, whereas clotrimazole can be used in the case of otitis caused by yeasts (M. pachidermatis), but at concentrations above $14 \mathrm{mg} / \mathrm{ml}$.
\end{abstract}

Key words: antimicrobial, antifungal, antibiotic, sensitivity

\section{INTRODUCCIÓN}

Entre los principales agentes etiológicos involucrados en los procesos de otitis externa en caninos se encuentran las levaduras, especialmente aquellas pertenecientes al género Malassezia. Estas son unas levaduras lipofílicas consideradas como microbiota cutánea y ótica normal, las cuales pueden convertirse en patógenas, de acuerdo con las condiciones microambientales del canal auditivo del hospedero y de las alteraciones que puedan ocurrir en el sistema inmune (Melchert et al., 2012).

Otros patógenos importantes en los procesos infecciosos óticos son las bacterias, tanto Gram positivas como Gram negativas. Algunas de ellas se pueden encontrar como microbiota normal del canal auditivo; sin embargo, pueden desarrollar patologías en casos de colonización masiva. Staphylococcus intermedius y Pseudomonas aeruginosa son las más asociadas a procesos infecciosos óticos, especialmente crónicos. Además, otras bacterias como Proteus mirabilis, Escherichia coli, Corynebacterium spp, Enterococcus spp y Streptococcus spp son causantes de otitis aunque en menor frecuencia (Angus, 2004).

El tratamiento para estas infecciones es con antimicrobianos elegidos con base a los hallazgos clínicos, y sin el uso de aislamientos microbiológicos y de pruebas de susceptibilidad antibacteriana (Mayanz, 2012), lo cual puede llevar a elegir un antibiótico inapropiado o desarrollar resistencia al medicamento (Machado, 2013).

La sensibilidad de los microorganismos a los antimicrobianos se mide mediante pruebas de sensibilidad o antibiograma, donde se evalúa la respuesta de un microorganismo a uno o varios antimicrobianos (Benavides et al., 2005). El nivel de efectividad de un antimicrobiano se determina por el método de Concentración Inhibitoria Mínima (CIM), midiendo la concentración más baja que inhibe el crecimiento visible de un microorganismo después de un periodo de incubación. La CIM antibiótica pueden ser determinada mediante métodos de dilución en caldo o en agar y su lectura se basa en métodos espectrofotométricos y halos de inhibición (Benavides et al., 2005).

En el presente estudio se evaluó la susceptibilidad de microorganismos aislados de perros con otitis, frente al principio activo de dos medicamentos de uso veterinario, producidos por una misma casa comercial. Además, se determinaron las dosis recomendadas para el principio activo del antifúngico. 


\section{Materiales y Métodos}

Se seleccionaron 27 perros con diagnóstico clínico de otitis en el Centro de Rescate y Adopción «Los Ángeles», institución que acoge perros abandonados. El diagnóstico clínico fue realizado por un médico veterinario especializado, docente de la Corporación Universitaria Lasallista, Caldas, Antioquia, Colombia, mediante observación directa. En la selección de los animales no se tuvo en cuenta la edad o raza.

Los casos de otitis fueron clasificados según la presencia de los signos clínicos como: Grado I: eritema, edema, calor; Grado II: eritema, edema, calor, dolor; Grado III: eritema, edema, calor, dolor leve, secreción; y Grado IV: eritema, edema, calor, dolor severo, estenosis del canal, secreción abundante de cualquier clase, inclinación de cabeza, fiebre.

Para la toma de muestras, se utilizó un aplicador que se introdujo suavemente en el pabellón auricular. Las muestras fueron colectadas con el medio de transporte Amies y llevadas al Laboratorio de Microbiología y Biotecnología de la Corporación Universitaria Lasallista para el aislamiento de microorganismos, excluyendo ácaros, virus y material particulado. Se hizo una siembra directa en medios selectivos Sabourand, Cetrimide, Baird Parker y MacConkey. Los medios se incubaron a $37^{\circ} \mathrm{C}$ por $24 \mathrm{~h}$ en condiciones aerobias. Luego se hizo caracterización morfológica y bioquímica de las colonias.

En el momento de disponerse de los resultados de las pruebas microbiológicas, se procedió al tratamiento de los canes con dos tipos de medicamentos clasificados como A y B, donde ambos tienen como principios activos a la gentamicina $(3 \mathrm{mg} / \mathrm{ml})$ y al clotrimazol $(10 \mathrm{mg} / \mathrm{ml})$, pero difieren en la cortisona (el medicamento A tiene betametasona y el $\mathrm{B}$ tiene mometasona). La mitad de los canes fueron tratados con el medicamento A y la otra mitad con el B. A los ocho días de la aplicación, se tuvo una espera de dos días y se realizó una segunda evaluación, tanto clínica como microbiológica, para determinar la efectividad del medicamento.

En la fase de tratamiento, tres perros murieron por causas ajenas al estudio. Cinco de los 24 perros que completaron el tratamiento continuaron con otitis. De estos, se aislaron y caracterizaron, tanto por métodos morfológicos como bioquímicos, Pseudomona aeruginosa $(\mathrm{n}=2)$, Staphylococcus intermedius $(\mathrm{n}=5)$ y levaduras del genero Malassezia $\mathrm{sp}(\mathrm{n}=8)$. Estos microorganismos se conservaron en crioviales con aceite mineral almacenados a $-80^{\circ} \mathrm{C}$.

Para los ensayos in vitro se utilizaron los dos aislados de $P$. aeruginosa, tres de $S$. intermedius y tres de Malassezia sp. Se cultivaron en medios líquidos selectivos para cada uno y se llevaron por patrón de McFarland a una concentración de $1 \times 10^{6}$ $\mathrm{UFC} / \mathrm{ml}$.

En las pruebas de CIM se emplearon los principios activos de los medicamentos utilizados en los tratamientos clínicos y en las dosis recomendadas por la casa comercial; es decir, gentamicina $(3 \mathrm{mg} / \mathrm{ml})$ y clotrimazol $(10 \mathrm{mg} / \mathrm{ml})$. Para esto, se preparó un stock de $3 \mathrm{ml}$ de cada fármaco en agua destilada estéril.

En las placas de ELISA se colocó $150 \mu 1$ de cada microorganismo y $150 \mu \mathrm{l}$ del fármaco (gentamicina para bacterias y clotrimazol para levaduras). Se hicieron dos repeticiones y tres réplicas de cada microorganismo; utilizando como control negativo los fármacos $(150 \mu \mathrm{l})$ más agua destilada estéril $(150 \mu \mathrm{l})$ y como control positivo los microorganismos en medio de cultivo $(150 \mu \mathrm{l})$ más agua destilada estéril. Las condiciones de crecimiento en el espectrofotómetro fueron de $35^{\circ} \mathrm{C} / 10 \mathrm{rpm} /$ $24 \mathrm{~h}$, donde cada $2 \mathrm{~h}$ se medía la cinética de crecimiento del microorganismo con una absorbancia (densidad óptica) de 660 nanómetros. 


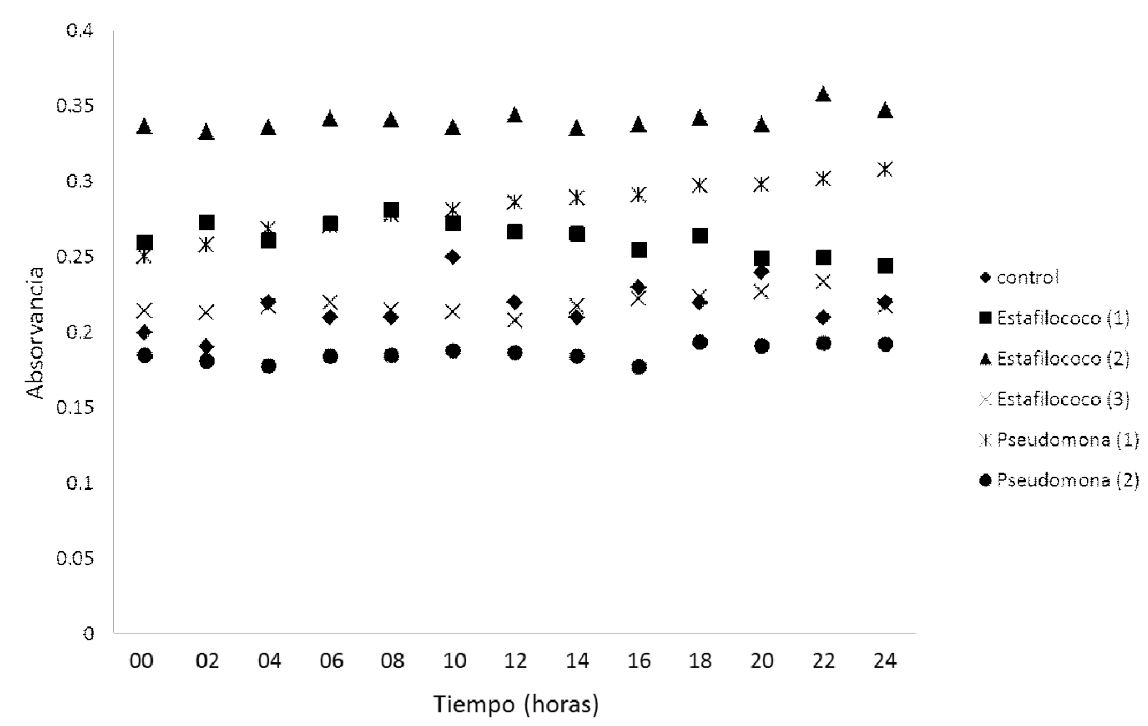

Figura 1. Evaluación de la gentamicina en dosis de $3 \mathrm{mg} / \mathrm{ml}$ frente a bacterias causantes de otitis crónicas en perros, mediante el método de Concentración Inhibitoria Mínima (CIM)

En el caso de las levaduras se empleó una evaluación adicional, consistente en una curva de evaluación de las concentraciones del antifúngico $(10,12,13,14$ y $15 \mathrm{mg} / \mathrm{ml})$, para determinar, mediante la cinética de crecimiento, la dosis recomendada contra las levaduras, ya que son las que sobrevivieron a la dosis sugerida por la casa comercial.

Todos los datos se tabularon y analizaron con el programa Statgraphics Centurion $X V$. Se hizo un análisis de varianza para determinar posibles diferencias estadísticas entre el crecimiento de los microorganismos.

\section{Resultados}

Como resultado de las 48 muestras de los 24 perros se obtuvieron 78 aislamientos, donde el $47 \%$ correspondió a estafilococos, $43 \%$ a levaduras y $8 \%$ a pseudomonas. No hubo diferencia estadística en la frecuencia de los grados de otitis ni entre el oído derecho o izquierdo. Asimismo, el prurito, la inflamación y el exudado oscuro fueron las prin- cipales manifestaciones clínicas, en tanto que la inapetencia no fue un signo evidente.

No hubo diferencia estadística entre los grados de otitis y los medicamentos utilizados, probablemente porque ambos medicamentos contienen los mismos principios activos, con excepción del corticoide, el cual no tiene efecto directo en el proceso infeccioso, sino en el proceso inflamatorio de la enfermedad.

La cinética de crecimiento bacteriano por efecto de la gentamicina mostró que todas las bacterias se vieron afectadas, con excepción de un $S$. intermedius (aislado 1), el cual presentó el mayor crecimiento respecto al control $(\mathrm{p}<0.05)$ (Fig. 1).

Las tres levaduras, aisladas de tres perros, fueron caracterizadas como Malassezia pachidermatis. Las evaluación de la efectividad de la dosis del antifúngico sugerida por la casa comercial demostró que no es efectiva para el control de la levadura (Fig. 2). Al evaluar la cinética de crecimiento de las le- 


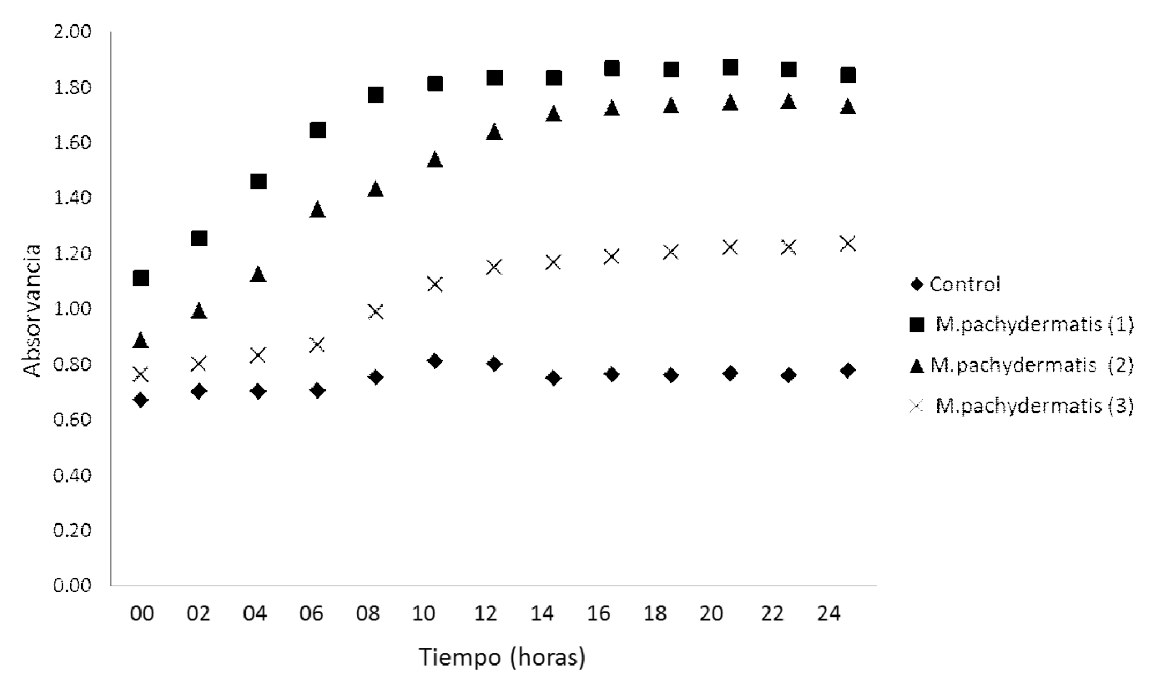

Figura 2. Evaluación del antifúngico clotrimazol en dosis de $10 \mathrm{mg} / \mathrm{ml}$ frente a levaduras causantes de otitis crónicas en perros, mediante el método de Concentración Inhibitoria Mínima (CIM)

vaduras a diferentes dosis de clotrimazol, se encontró que las levaduras mostraron mayor sensibilidad a partir de las dosis de $14 \mathrm{mg} / \mathrm{ml}$.

\section{Discusión}

La otitis externa es la enfermedad más común en el canal auditivo de perros y normalmente su etiología es referida a organismos como Staphylococcus intermedius y Malassezia pachydermatis, siendo este último el principal agente etiológico de la otitis externa (Graham-Mize y Rosser, 2004; Kaneko et al., 2007; Petrov y Mihaylov, 2008; Peano et al., 2012). Los resultados del presente estudio corroboran estos hallazgos, donde se aislaron ocho levaduras caracterizadas como M. pachidermatis.

Oliveira et al. (2008) reportaron una asociación frecuente entre $M$. pachydermatis y Staphylococcus spp en casos de otitis canina; sin embargo, Carvalho (2005) asegura que agentes como $P$. aeruginosa, Streptococcus spp y Staphylococcus spp son determinantes en la aparición de la enfermedad. De hecho, los hallazgos en la presente investigación ratifican que $S$. intermedius y $P$. aeruginosa fueron causantes de la otitis externas de varios de los perros tratados durante la investigación.

Sánchez et al. (2011) reportaron que las bacterias Gram positivas y negativas, aisladas de pacientes con otitis, fueron susceptibles a una serie de antibióticos, incluyendo las quinolonas, aminoglucósidos, cefalosporinas y penicilinas combinadas con inhibidores de las betalactamasas, mientras que las penicilinas, sulfas, tetraciclinas, lincosamidas y macrólidos fueron los antimicrobianos de menor efectividad. En este sentido, el aminoglucósido gentamicina, utilizado en el presente estudio, sigue siendo uno de los antibióticos de elección para el control del crecimiento de los microorganismos causantes de la otitis.

En las infecciones por levaduras, $M$. pachydermatis es considerada una de las principales causas de otitis, por ser un pató- 
geno oportunista, comensal obligado de la piel y del conducto auditivo externo, especialmente de carnívoros. En determinadas circunstancias, su presencia se relaciona con cambios en los mecanismos inmunológicos de su hospedador que regulan la colonización y su posterior carácter infectivo (Bensignor y Grandemange, 2006).

La dosis de clomitrazol recomendada por la casa comercial $(10 \mathrm{mg} / \mathrm{ml})$ resultó inefectiva, debiendo incrementarse hasta 15 $\mathrm{mg} / \mathrm{ml}$ para obtener buenos efectos, según los resultados del presente estudio. Se ha reportado que $M$. pachydermatis y $M$. furfur son las levaduras menos sensibles a los azoles y la resistencia a estos agentes está determinada por la producción de $14 \alpha$-demetilasas (Garau et al., 2003), las cuales generan cambios en la composición de los esteroles y fosfolípidos de la membrana celular fúngica que conlleva a una disminución en la adquisición de los azoles (Guillot y Bond, 1999; García, 2005).

\section{Conclusiones}

- Para el control de los agentes bacterianos causantes de otitis en caninos, la gentamicina en una dosis de $3 \mathrm{mg} / \mathrm{ml}$, produce una adecuada inhibición del crecimiento de los microorganismos.

- El clotrimazol podría emplearse en el caso de otitis causadas por levaduras (M. pachidermatis), pero en concentraciones superiores a $14 \mathrm{mg} / \mathrm{ml}$.

\section{Literatura Citada}

1. Angus JC. 2004. Otic cytology in health and disease. Vet Clin North Am Small Anim Pract 34: 411-424.

2. Benavides-Plascencia L, AldamaOjeda A, Vázquez H. 2005. Vigilancia de los niveles de uso de antibióticos y perfiles de resistencia bacteriana en hos- pitales de tercer nivel de la Ciudad de México. Salud Pública Méx 47: 219-226.

3. Bensignor E, Grandemange E. 2006. Comparison of an antifungal agent with a mixture of antifungal, antibiotic and corticosteroid agents for the treatment of Malassezia species otitis in dogs. Vet Rec 158: 193-195. doi: 10.1136/ vr.158.6.193

4. Carvalho CBM, Oliveira LC, Medeiros CMO, Silva ING Monteiro AJ, Leite CAL. 2005. Susceptibilidade a antimicrobianos de bacterias isoladas de otite externa em cães. Arq Bras Med Vet Zootec 57: doi: 10.1590/S0102

5. Garau M, Pereiro M Jr, del Palacio A. 2003. In vitro susceptibilities of Malassezia species to a new triazole, albaconazole (UR-9825), and other antifungal compounds. Antimicrob Agents Chemother 47: 2342-2344. doi: 10.1128/AAC.47.7.2342-2344.2003

6. García JP. 2005. Mecanismos de resistencia a los antifúngicos. Rev Esp Quimioter 18: 29-30.

7. Graham-Mize CA, Rosser EJ Jr. 2004. Comparison of microbial isolates and susceptibility patterns from external ear canal of dogs with otitis externa. J Am Anim Hosp Assoc 40: 102-108.

8. Guillot J, Bond R. 1999. Malassezia pachydermatis. A review. Med Mycol 37:295-306.

9. Kaneko T, Makimura K, Abe M, Shiota $R$, Nakamura Y, Kano R, Hasegawa; $A$, et al. 2007. Revised culture-based system for identification of Malassezia species. J Clin Microbiol 14:3737-3742. doi: 10.1128/JCM.01243-07

10. Machado VMMC. 2013. Otite externa canina: estudo preliminar sobre a otalgia e factores associados. Maestría en Medicina Veterinaria. Lisboa, Portugal: Universidade Lusófona de Humanidades e Tecnologias. $62 \mathrm{p}$.

11. Mayanz VB. 2012. Pioderma en el canino. REDVET 13(3). [Internet]. Disponible en: http://www.veterinaria.org/ revistas/redvet/n030312/031201.pdf 
12. Melchert A, Jefery ABS, Giuffrida R. 2012. Avaliações citológicas em otites caninas por Malassezia spp: estudo retrospectivo. Colloquium Agrariae 7(2): 27-34.

13. Oliveira LC, Leite CA, Brilhante RS, Carvalho CB. 2008. Comparative study of the microbial profile from bilateral otitis externa. Can Vet J 49: 785-788.

14. Peano A, Beccati M, Chiavassa E, Pasquetti M. 2012. Evaluation of the antifungal susceptibility of Malassezia pachydermatis to clotrimazole, miconazole and thiabendazole using a modified CLSI M27-A3 microdilution method. Vet Dermatol 23: 131-135. doi: 10.1111/j.1365-3164.2011.01025.x.

15. Petrov V, Mihaylov G. 2008. Malassezia pachydermatis - Etiology and clinical findings in canine external otitis - therapeutic approaches. Trakia J Sci 6 (Suppl 1): 123-126.

16. Sánchez $R$, Calle S, Falcón N, Pinto C. 2011. Aislamiento bacteriano en casos de otitis canina y su susceptibilidad antibiótica. Rev Inv Vet Perú 22: 161-166. 